\title{
Analyzing Untreated Alcohol Use Disorder and Factors Related to Help-Seeking Status
}

\author{
Soo Jung Rim ${ }^{1}$, MinKyung Jo ${ }^{1}$, Junghyun H. Lee ${ }^{2}$, and Subin Park ${ }^{3 凶}$ \\ ${ }^{1}$ Department of Research Planning, National Center for Mental Health, Seoul, Republic of Korea \\ ${ }^{2}$ National Center for Disaster and Trauma, Seoul, Republic of Korea \\ ${ }^{3}$ Mental Health Research Institute, National Center for Mental Health, Seoul, Republic of Korea
}

\begin{abstract}
Objective Alcohol use disorder (AUD) is a global burden but is also one of the most undertreated mental disorders. This study estimates the treatment rate (i.e., help-seeking status) of high-risk drinkers and factors associated with not receiving treatment in Korea using nationally representative National Health Insurance Service-National Sample Cohort (NHIS-NSC) data.

Methods Among 1,025,340 participants, we eliminated those under 20 and those who did not respond to the question regarding alcohol consumption. High-risk drinkers were classified based on their frequency and quantity of alcohol consumption (4 or more drinks for women, 5 or more drinks for men per day on average). In total, 32,225 high-risk drinkers were checked for an actual AUD diagnosis to see their treatment-seeking status.

Results Among 32,225 high-risk drinkers, only 1.24\% had an AUD diagnosis, showing that over 98\% of those who could be diagnosed with AUD are not seeking treatment. Factors associated with not seeking treatment were female sex, BMI higher or equal to 18.6, former smoker, middle-aged, and Charlson Comorbidity Index (CCI).

Conclusion Our data show that many high-risk drinkers do not seek treatment in Korea. Experts, policymakers, and clinicians should promote help-seeking behavior for AUD and pay close attention to those at risk of not receiving treatment for AUD.
\end{abstract}

Psychiatry Investig 2021;18(10):936-942

Keywords Alcoholism; Help-seeking behavior; Republic of Korea.

\section{INTRODUCTION}

Alcohol use disorder (AUD) is a global burden, with approximately 100 million estimated cases, the highest among all substance use disorders, as of $2016 .{ }^{1}$ Moreover, AUD contributes to about $4 \%$ of the global burden of disease. ${ }^{2}$ Specifically, the 12-month prevalence estimate of AUD, among those age 15 or older in 2016, was $13.9 \%$ for Korea, which is much higher than that of the AUD of Western Pacific WHO-affiliated countries $(4.7 \%)^{3}$

The effect of alcohol consumption on an individual's health depends on the amount of alcohol consumption. ${ }^{4}$ With low-

Received: April 5, 2021 Revised: June 26, 2021

Accepted: July 29, 2021

$\triangle$ Correspondence: Subin Park, MD, PhD

Mental Health Research Institute, National Center for Mental Health, 127 Yongmasan-ro, Gwangjin-gu, Seoul 04933, Republic of Korea

Tel: +82-2-2204-0103, Fax: +82-2-2204-0393, E-mail: subin-21@hanmail.net

(c) This is an Open Access article distributed under the terms of the Creative Commons Attribution Non-Commercial License (https://creativecommons.org/licenses/by$\mathrm{nc} / 4.0$ ) which permits unrestricted non-commercial use, distribution, and reproduction in any medium, provided the original work is properly cited. dose consumption, there are possible beneficial effects, such as lowering the risk of ischemic stroke, sudden cardiac death, osteoporosis, diabetes mellitus, cancer, and obesity. ${ }^{4-6}$ However, heavy alcohol consumption is associated with clear negative consequences: higher risk of diabetes, ${ }^{7}$ coronary and peripheral artery disease, stroke, hypertension, and so on. ${ }^{8}$ Additionally, alcohol hangovers are associated with impaired cognitive functions, ${ }^{9}$ and current heavy alcohol consumption has lasting consequences on the individual's cognitive function (e.g., learning, memory). ${ }^{10}$ Moreover, AUD is highly comorbid with other psychiatric disorders, and being comorbid with AUD further increases the risk of suicide mortality of individuals with a preceding psychiatric disorder. ${ }^{11}$

Despite these detrimental effects of heavy alcohol consumption, AUD is undertreated. ${ }^{12}$ Previous research studies indicate that less than a quarter of those who are eligible for AUD diagnosis, receives treatment. ${ }^{13,14} \mathrm{~A}$ review study found that the treatment gap for alcohol abuse and alcohol dependence was the greatest among people with mental disorders: median $78.1 \%$ (ranging from $49.4 \%-96 \%$ ). ${ }^{15}$ Considering the detri- 
mental effects of AUD and its low treatment rate, it is important to find factors related to not seeking help and to provide appropriate interventions.

However, the majority of previous studies such as those by Cohen et al. ${ }^{14}$ and Kessler et al., ${ }^{16}$ only considered treatment utilization by actual patients and relied on the patient's response to measure treatment utilization. It is important to investigate the characteristics of those who are eligible for AUD treatment but do not seek treatment, by using diagnostic records. Therefore, this study used a nationally representative cohort data to investigate treatment utilization (diagnosis record) of those with provisional AUD (including those with an AUD diagnosis and those who could be considered for an AUD diagnosis but did not seek treatment) and to explore the factors associated with not seeking treatment.

\section{METHODS}

\section{Data source}

South Korea achieved universal health coverage in 1989 through National Health Insurance (NHI). In 2000, NHI consolidated all related societies under a single payer, National Health Insurance Service (NHIS). Further, NHIS built a nationally representative database for research and policy development-the National Health Insurance Service-National Sample Cohort (NHIS-NSC). Participants from the national health insurance service-national sample cohort (NHIS-NSC) data (2002 to 2013) were included in the analysis. This study was approved by the Institutional Review Board (IRB) of the National Center for Mental Health, Ministry of Health and Welfare (116271-2020-01). Informed consent was waived since NHIS-NSC data is comprised of administrative data or they claim data which are routinely collected. The NHIS-NSC is comprised of 1,025,340 individuals, or $2 \%$ of the whole nation. To structure the database, 18 strata were built according to age group, sex, eligibility status, and income level; then, for each stratum, systematic sampling was done, maintaining the designated sampling rate (i.e., 2.2\%). Additional information about NHIS-NSC can be found elsewhere. ${ }^{17}$

Participants in the cohort were followed up for about 11 years, until 2013. Each year, there were inevitable dropouts due to death or emigration; to address this, about 9,000 infants aged 0 were newly added to the cohort. NHIS-NSC includes four databases (DBs). The qualification DB comprises the general information of health insurance subscribers and medicare recipients, such as sex, age, location, type of subscription, etc. The treatment $D B$ is composed of information such as treatment and medication of the subjects. The medical checkup $D B$ includes data regarding medical check-up as well as behavior and habitual data. Last, the clinic $D B$ is composed of information on the clinic that subjects visit, such as equipment, type, location, etc. We identified those who could be considered for AUD through the medical check-up DB, which includes a self-questionnaire about the individual's health behavior, including alcohol consumption. Help-seeking behavior was derived from the individual's treatment DB. More details about the cohort can be found elsewhere. ${ }^{17}$

\section{Participant selection}

Health checkups were included under the insurance benefits of the NHIS. There are three different types of checkups, categorized by age: infant, youth, and adult. The adult checkups include general screening, cancer screening, and health screening. Among various screening items, we utilized self-report about the participants' lifestyle, specifically, alcohol consumption. Within the NHIS-NSC, those who were under 20 and those who did not give a response to the question regarding alcohol consumption were excluded from the analysis. Among those who remained, we categorized participants as "high-risk drinkers" or not, based on their frequency and quantity of alcohol consumption in the past month. For frequency, the question was "How often did you drink alcohol in the past month?" The responses options were 1) do not drink, 2) 2-3 times a month, 3) once or twice a week, 4) 3-4 times a week, 5) almost every day. The quantity of alcohol consumption was based on the following question: "On one occasion, how much alcohol do you usually drink?" The response options were 1) around half a bottle or less of soju or equivalent, 2) around one bottle of soju or equivalent, 3) around one and a half bottles of soju or equivalent, or 4) around more than two bottles of soju or equivalent (soju is the most popular Korean traditional liquor with about $20 \%$ alcohol content). The criteria for "high-risk drinkers" were based on previous literature: women who have 4 or more drinks per day and men who have 5 or more drinks per day on average. ${ }^{10,18}$ One average soju bottle comprises 7 drinks. Ultimately, 32,225 participants were classified as "high-risk drinkers" and were included in the analysis (Figure 1). After classifying high-risk drinkers, we then checked whether they had received a diagnosis of AUD (F10) based on the ICD-10 code during the follow-up period. However, since we only considered the quantity and frequency of alcohol consumption and did not consider how alcohol consumption interferes with individual's daily life (one of the diagnostic criteria in DSM-5), not all high-risk drinkers may actually have AUD.

\section{Variables}

We selected variables that could be related to AUD treatment status from the NHIS-NCS. For those who had received a diagnosis of AUD, we merged the most recent demographic 


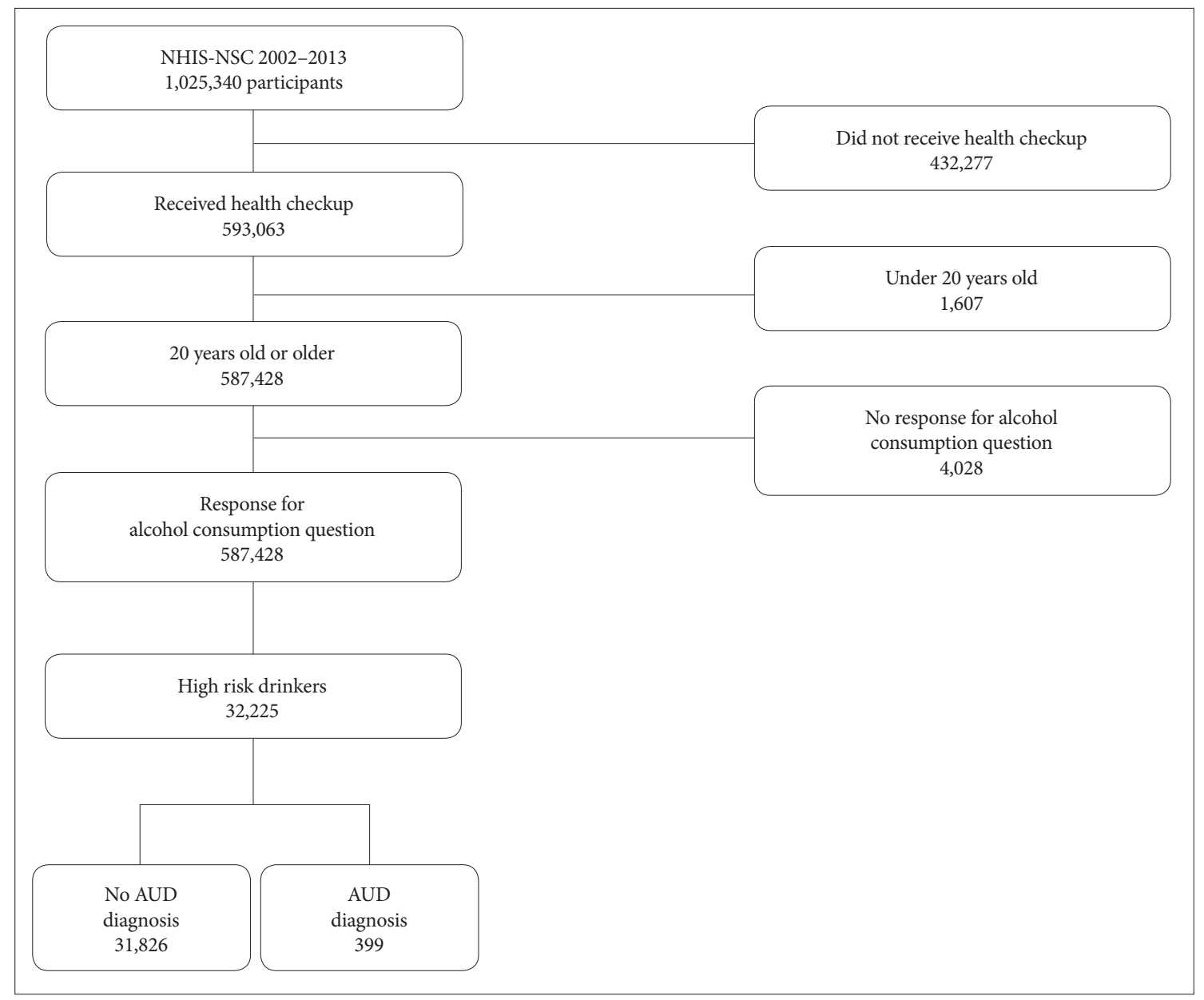

Figure 1. Participant selection process. AUD, alcohol use disorder.

and health check-up data preceding AUD diagnosis; for those without an AUD diagnosis, we utilized the latest health checkup data. The variables included in the analysis were as follows: sex, age, body mass index (BMI), current smoking status (never smoked, former smoker, current smoker), physical activity per week (none, 1-4 times, 5-7 times), income level (medical aid, low, middle, and high), Charlson Comorbidity Index (CCI), and diagnosis of hypertension or diabetes. Sex was determined based on how individuals were registered by the insurance system (i.e., male or female). Age was divided into three ranges: $20-39,40-69$, and 70 or older. Current smoking status and physical activity were self-rated by participants using the (respective) questions, "How much do you smoke?" and "How many times a week do you exercise to the point where you sweat?" Current smoking status was categorized based on the response choices of 1) never smoked, 2) smoked in the past but not anymore, and 3) smoke currently, while physical activity was determined through 1) never, 2) 1-2 times, 3) 3-4 times, 4) 5-6 times, and 5) almost every day. Income level was based on the participant's contribution level (payment) to the NHI. Thus, higher the income, higher the contribution level, where a contribution level of 0 indicated the lowest income level (receiving medical aid beneficiaries), while 10 indicated highest income and contribution level. Therefore, contribution levels were classified as 0 (medical aid), 1-3 (low income), 4-6 (middle income), and 7-10 (high income). CCI categorizes the individual comorbid condition (which may influence mortality risk). ${ }^{19}$ Each condition (e.g., hypertension, acquired immune deficiency syndrome [AIDS]) has its own severity level, where 0 indicates no comorbid conditions and higher scores indicate multiple comorbid conditions that may increase the fatality risk. BMI, CCI, and diagnosis of hypertension and diabetes were derived or calculated from participants' health records.

\section{Statistical analysis}

We classified participants as "high-risk drinkers" based on their responses regarding their drinking pattern (average drinks consumed per day for each participant). We then compared demographics, health management behavior, and comor- 
bid physical disease data between the treatment-seeking group and the no-treatment-seeking group through chi-squared analysis. Multiple logistic regression was performed to investigate factors associated with patients not receiving treatment (diagnosis of AUD) even if those patients could qualify as having AUD. P-values of $<0.01$ were considered statistically significant. All statistical analyses were performed with SAS software version 9.4 (SAS Institute Inc., Cary, NC, USA).

\section{RESULTS}

This study has estimated the number of high-risk drinkers who have a high possibility of meeting the diagnostic criteria for AUD and their treatment rate. We also identified characteristics associated with not seeking treatment. As shown in Figure 1, there were 32,225 (about 5.5\%) participants who could be considered high-risk drinkers. Among these individuals, only 399 (1.24\%) were diagnosed as AUD, that is, 31,826 (98.76\%) did not receive treatment. Table 1 shows the characteristics of participants based on AUD diagnosis status.

Table 2 shows the risk of not receiving treatment by sex, age group, BMI, smoking status, physical activity (per week), and CCI. Specifically, female sex $(\mathrm{AOR}=1.677,95 \% \mathrm{CI}=1.074$, 2.618), BMI 18-22.9 (AOR=1.614, 95\% CI=1.023, 2.546), BMI $\geq 23$ ( $\mathrm{AOR}=2.794,95 \% \mathrm{CI}=1.1771,4.408)$, and being a former smoker $(\mathrm{AOR}=2.528,95 \% \mathrm{CI}=1.724,3.708)$ were associated with higher risk of not receiving help for AUD, and being middle-aged $(\mathrm{AOR}=0.622,95 \% \mathrm{CI}=0.474,0.815)$, having $\mathrm{CCI}$ of $2(\mathrm{AOR}=0.527,95 \% \mathrm{CI}=0.403,0.690)$, and $\mathrm{CCI} \geq 3(\mathrm{AOR}=$ $0.436,95 \% \mathrm{CI}=0.323,0.587)$ were associated with lower risk of not receiving help after adjusting for related variables.

\section{DISCUSSION}

Our findings indicated that 32,225 out of 587,428 participants (i.e., those who responded about alcohol consumption in the questionnaire) were classified as high-risk drinkers. This suggests that about $5.5 \%$ of them are high-risk drinkers, who could be considered to have AUD. This percentage is slightly higher than the one-year prevalence rate of $3.5 \%$ for AUD according to The Survey of Mental Disorders in Korea 2016. ${ }^{20}$ This study classifies individuals as 'high-risk drinkers' using two factors: frequency and quantity of alcohol consumption. In contrast, the Survey of Mental Disorders in Korea utilized the Korean version of the Composite International Diagnostic Interview, ${ }^{21}$ which applied full diagnostic criteria. This may explain the discord between the two studies' prevalence rates.

Moreover, among 32,225 high-risk drinkers, only 399 have been diagnosed with AUD. This means that only $1.24 \%$ of high-risk drinkers have received treatment. This is broadly
Table 1. Participant characteristics

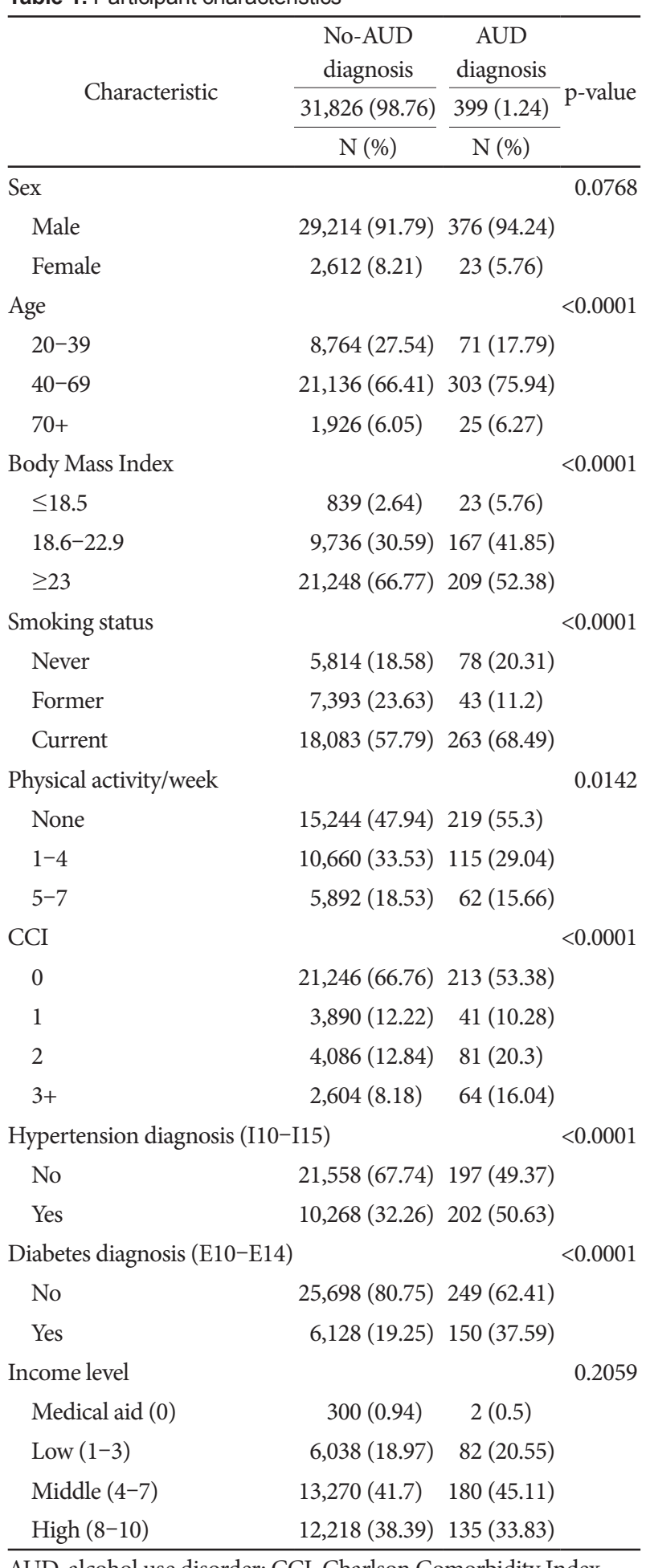

AUD, alcohol use disorder; CCI, Charlson Comorbidity Index

consistent with previous studies showing low treatment rates (i.e., less than 25\%) for alcohol use disorders; $13,{ }^{22}$ however, at only $1.24 \%$ treated, the treatment gap in Korea seems to be much more problematic. There are several explanations for the low treatment rate of AUD in general. First of all, there is a ten- 
Table 2. Risk of not receiving treatment by sociodemographic variables, health behavior, and comorbid disease

\begin{tabular}{|c|c|c|c|c|c|c|}
\hline Characteristic & OR & LCI & UCI & AOR & LCI & UCI \\
\hline \multicolumn{7}{|l|}{ Sex } \\
\hline Male (ref) & 1 & & & 1 & & \\
\hline Female & 1.462 & 0.958 & 2.231 & 1.677 & 1.074 & 2.618 \\
\hline \multicolumn{7}{|l|}{ Age } \\
\hline 20-39 (ref) & 1 & & & 1 & & \\
\hline $40-69$ & 0.565 & 0.436 & 0.733 & 0.622 & 0.474 & 0.815 \\
\hline $70+$ & 0.624 & 0.395 & 0.987 & 0.946 & 0.574 & 1.558 \\
\hline \multicolumn{7}{|l|}{ Body Mass Index } \\
\hline$\leq 18.5$ (ref) & 1 & & & 1 & & \\
\hline $18.6-22.9$ & 1.598 & 1.028 & 2.485 & 1.614 & 1.023 & 2.546 \\
\hline$\geq 23$ & 2.787 & 1.802 & 4.31 & 2.794 & 1.771 & 4.408 \\
\hline \multicolumn{7}{|l|}{ Smoking status } \\
\hline Never (ref) & 1 & & & 1 & & \\
\hline Former & 2.306 & 1.587 & 3.351 & 2.528 & 1.724 & 3.708 \\
\hline Current & 0.922 & 0.715 & 1.19 & 1.026 & 0.783 & 1.344 \\
\hline \multicolumn{7}{|c|}{ Physical activity (per week) } \\
\hline None (ref) & 1 & & & 1 & & \\
\hline $1-4$ & 1.332 & 1.061 & 1.671 & 1.178 & 0.931 & 1.492 \\
\hline $5-7$ & 1.365 & 1.028 & 1.813 & 1.167 & 0.874 & 1.559 \\
\hline \multicolumn{7}{|l|}{ CCI } \\
\hline 0 (ref) & 1 & & & 1 & & \\
\hline 1 & 0.951 & 0.68 & 1.331 & 0.915 & 0.65 & 1.289 \\
\hline 2 & 0.506 & 0.391 & 0.655 & 0.527 & 0.403 & 0.69 \\
\hline $3+$ & 0.408 & 0.307 & 0.541 & 0.436 & 0.323 & 0.587 \\
\hline
\end{tabular}

CCI, Charlson Comorbidity Index; OR, odds ratios; LCI, low confidence interval; HCI, high confidence interval; AOR, adjusted odds ratios; ref, reference value

dency for drinkers to underestimate the amount they consume. ${ }^{23}$ Another related factor is high stigma towards AUD: a previous study found that AUD patients with higher stigma towards AUD were less likely to seek help, meaning that stigma towards patients with AUD could interfere with patients' help-seeking behavior. ${ }^{18}$ Moreover, according to a recent review study, alcoholism can go into remission without formal treatment, which could explain the low treatment rate. ${ }^{24} \mathrm{How}-$ ever, the same review study also states that formal treatment is still important, since it is impossible at this stage to predict who will or will not experience spontaneous remission.

In addition to the aforementioned explanations for the low treatment rate of AUD, the unique drinking culture of Korea could be a factor that reduces treatment rate. Typically, Koreans' drinking behavior is characterized as "social drinking," and when they drink, there is a culture where individuals take a "one-shot" drink of alcohol rather than a sip and then hand the empty glass over to the next person to take a "one-shot".
This culture may lead individuals to drink more alcohol. In line with this, Korea has a tendency to be lenient towards alcohol consumption. ${ }^{26}$ Furthermore, drinking alcohol is an important element not only in social gatherings but also in professionaly fields. Korean workers believe that alcohol is an important component in building and maintaining relationships with their coworkers; ${ }^{27}$ as a consequence, the social costs associated with alcohol (loss of productivity, health care costs, etc.) account for about $2 \%$ of Korea’s GDP ${ }^{28}$ However, despite the consequences of risky drinking, alcohol consumption in Korea has been continuously increasing for the past 40 years. ${ }^{26}$ It seems like alcohol consumption is an important means of maintaining social and work life in Korea and that Korean society's lenient attitude towards alcohol consumption may blind individuals to the consequences of risky drinking behavior. The government, policymakers, and clinicians should put in more effort to decrease alcohol consumption and increase treatment utilization for people with AUD and high-risk drinkers.

There was a sex difference in help-seeking behavior for AUD, with women having a higher risk of not receiving help. This is in line with previous studies that have found that women are less likely than men to receive help for alcohol problems. ${ }^{29-31}$ Furthermore, previous literature states that women have unique barriers to seeking treatment compared to men: ${ }^{32}$ greater economic barriers and family responsibilities, stigma, and social disapproval. ${ }^{33,34}$ This shows that there need to be sex-specific strategies when identifying those who need help with AUD.

Being a former smoker was associated with higher risk of not receiving help than never having smoked. There are concerns that smoking cessation leads to higher risk of other substance use. ${ }^{35}$ However, according to a recent review study, smoking cessation was not associated with higher risk of other substance use; in fact, almost $50 \%$ of the studies showed solely positive effects of smoking cessation. ${ }^{36}$ Furthermore, another recent study states that smoking cessation does not affect changes in alcohol consumption. ${ }^{37}$ Rather, smoking cessation may be associated with stress levels: In our study, those who had successfully quit smoking had lower stress levels compared to those who did not attempt to quit smoking $(\mathrm{OR}=$ $0.87,95 \% \mathrm{CI}=0.86-0.89)$. Moreover, those who attempted but failed to quit smoking had higher stress than those who did not attempt to quit ( $\mathrm{OR}=1.11,95 \% \mathrm{CI}=1.09-1.14) .{ }^{38}$ Combining these results, it is possible to infer that smoking cessation may not be related to a person's amount of alcohol consumption or other substance use problems, but that it might affect the individual's psychological distress. Therefore, former smokers may not feel the need to receive help for AUD, since they might have lower levels of psychological distress.

BMI less than 18.6 was associated with receiving treatment for AUD. This suggests that high-risk drinkers who have a nor- 
mal BMI or who are obese have a higher risk of not receiving help. According to a previous study, the relationship between BMI and mortality has a j-shaped relationship, suggesting that being underweight as well as overweight could be associated with mortality. ${ }^{39}$ Moreover, the all-cause mortality was lowest among those with normal BMI (between 20 and 24.9). Therefore, those with normal BMI may not seek help, since they have the lowest risk of health conditions and mortality, while those with low BMI may seek treatment, since they have more complicated health issues. Moreover, previous research ${ }^{40}$ indicates that higher BMI is associated with more perceived barriers in weight-loss treatment seeking and specifically with the stigma-related barrier (i.e., "I am afraid people will treat me unfairly or badly"); such a perceived stigma may have a negative impact on treatment-seeking for AUD as well as weightloss treatment-seeking.

Respondents with more comorbid diseases had a lower risk of not receiving diagnosis for AUD (i.e., those with more comorbid diseases seek help more compared to those with less comorbid diseases). According to previous studies, those with risky drinking problems only seek treatment when they have substantial difficulties in their life. ${ }^{41,42}$ This could be related to the fact that patients usually get diagnosed with AUD while being admitted for a physical disease (e.g., liver or gastrointestinal disease). ${ }^{2}$ Also, those with AUD have a high risk of having other, comorbid psychiatric disorders. ${ }^{43}$ Therefore, an evaluation for AUD is necessary for those who visit the hospital for a mental illness or alcohol-related physical condition.

Middle-aged respondents showed lower risk of not seeking treatment for AUD compared to younger ones. This finding is in line with previous studies showing treatment-seeking behavior increasing with age. ${ }^{14,31,44,45}$ For the younger age group, perceived stigma or fear that being diagnosed with a substance abuse disorder will negatively influence their career could limit them from seeking help. ${ }^{14}$ More efforts to encourage help-seeking behavior in younger people are needed.

Despite the meaningful findings of this study, there are also several limitations. First, we defined high-risk drinkers (provisional AUD) based only on the participants' frequency and quantity of alcohol consumption; that is, we did not consider how alcohol consumption affects each individual's daily life functioning, which is another diagnostic criterion for AUD. Second, since we conducted a retrospective cohort study, there may have been some limitation on the data that we could utilize for the purpose of this study. Future studies need to conduct prospective cohort research to get a clearer view of the relationship between high-risk drinkers, help-seeking behavior, and related factors. Another limitation is that since the frequency and quantity of alcohol consumption were based on participants' self-report, there may have been bias in their re-

sponses.

In conclusion, this study has estimated the treatment rate of those at high risk for AUD in Korea. Overall, the treatment rate of high-risk drinkers is quite low, and there need to be more efforts to emphasize the risks of heavy alcohol consumption. Moreover, we investigated the factors associated with not seeking treatment; efforts are needed to motivate individuals to seek treatment for AUD based on these factors. Researchers, policymakers, and related professionals should use this study to plan prevention and treatment efforts for AUD.

\section{Availability of Data and Material}

The datasets generated or analyzed during the current study are available in the National Health Insurance Sharing Service repository, [https:// nhiss.nhis.or.kr/bd/ab/bdaba013eng.do].

\section{Conflicts of Interest}

Subin Park, a contributing editor of the Psychiatry Investigation, was not involved in the editorial evaluation or decision to publish this article. All remaining authors have declared no conflicts of interest.

\section{Author Contributions}

Conceptualization: all authors. Data curation: all authors. Formal analysis: MinKyung Jo. Methodology: MinKyung Jo, Subin Park. Writing—original draft: Soo Jung Rim. Writing — review and editing: Soo Jung Rim, Junghyun H. Lee, Subin Park. Approval of final manuscript: all authors.

\section{ORCID iDs}

Soo Jung Rim MinKyung Jo

Junghyun H. Lee

Subin Park

\begin{abstract}
https://orcid.org/0000-0003-4938-4116 https://orcid.org/0000-0002-4208-0024 https://orcid.org/0000-0001-9116-4593 https://orcid.org/0000-0002-4623-9899
\end{abstract}

\section{Funding Statement}

This work was supported by the National Center for Mental Health, Republic of Korea, under a clinical research grant [number 2020-01].

\section{REFERENCES}

1. Degenhardt L, Charlson F, Ferrari A, Santomauro D, Erskine H, Mantilla-Herrara A, et al. The global burden of disease attributable to alcohol and drug use in 195 countries and territories, 1990-2016: a systematic analysis for the Global Burden of Disease Study 2016. Lancet Psychiatry 2018;5:987-1012.

2. Connor J, Haber P, Hall WD. Alcohol use disorders. Lancet 2016;387: 988-998.

3. WHO. Alcohol Consumption: Levels and Patterns-Republic of Korea. Geneva: WHO; 2018. Available at: https://www.who.int/substance_ abuse/publications/global_alcohol_report/profiles/kor.pdf.

4. Reynolds K, Lewis BL, Nolen JDL, Kinney GL, Sathya B, He J. Alcohol consumption and risk of stroke: A meta-analysis. JAMA 2003;289:579588.

5. Chiuve S, Rimm E, Mukamal K, Rexrode K, Stampfer M, Manson J, et al. Light-to-moderate alcohol consumption and risk of sudden cardiac death in women. Heart Rhythm 2010;7:1374-1380.

6. Rubin E. To drink or not to drink: that is the question. Alcohol Clin Exp Res 2014;38:2889-2892.

7. Howard AA, Arnsten JH, Gourevitch MN. Effect of alcohol consumption on diabetes mellitus: a systematic review. Ann Intern Med 2004;140:211219.

8. Fernández-Solá J. Cardiovascular risks and benefits of moderate and 
heavy alcohol consumption. Nat Rev Cardiol 2015;12:576-578.

9. Gunn C, Mackus M, Griffin C, Munafo MR, Adams S. A systematic review of the next-day effects of heavy alcohol consumption on cognitive performance. Addiction 2018;113:2182-2193.

10. Woods AJ, Porges EC, Bryant VE, Seider T, Gongvatana A, Kahler CW, et al. Current heavy alcohol consumption is associated with greater cognitive impairment in older adults. Alcohol Clin Exp Res 2016;40:24352444.

11. Park S, Rim SJ, Jo M, Lee MG, Kim CE. Comorbidity of alcohol use and other psychiatric disorders and suicide mortality: data from the South Korean National Health Insurance Cohort, 2002 to 2013. Alcohol Clin Exp Res 2019;43:842-849.

12. Rehm J, Anderson P, Barry J, Dimitrov P, Elekes Z, Feijão F, et al. Prevalence of and potential influencing factors for alcohol dependence in Europe. Eur Addict Res 2015;21:6-18.

13. Hasin DS, Stinson FS, Ogburn E, Grant BF. Prevalence, correlates, disability, and comorbidity of DSM-IV alcohol abuse and dependence in the United States: results from the National Epidemiologic Survey on Alcohol and Related Conditions. Arch Gen Psychiatry 2007;64:830-842.

14. Cohen E, Feinn R, Arias A, Kranzler H. Alcohol treatment utilization: findings from the National Epidemiologic Survey on Alcohol and Related Conditions. Drug Alcohol Depend 2007;86:214-221.

15. Kohn R, Saxena S, Levav I, Saraceno B. The treatment gap in mental health care. Bull World Health Org 2004;82:855-866.

16. Kessler RC, Chiu WT, Demler O, Walters EE. Prevalence, severity, and comorbidity of twelve-month DSM-IV disorders in the National Comorbidity Survey Replication (NCS-R). Arch Gen Psychiatry 2005;62: 617-627.

17. Lee J, Lee JS, Park SH, Shin SA, Kim K. Cohort profile: the National Health Insurance Service-National Sample Cohort (NHIS-NSC), South Korea. Int J Epidemiol 2016;46:e15.

18. Keyes KM, Hatzenbuehler ML, McLaughlin KA, Link B, Olfson M, Grant BF, et al. Stigma and treatment for alcohol disorders in the United States. Am J Epidemiol 2010;172:1364-1372.

19. Charlson ME, Pompei P, Ales KL, MacKenzie CR. A new method of classifying prognostic comorbidity in longitudinal studies: development and validation. J Chron Dis 1987;40:373-383.

20. Hong J, Lee DW, Ham B, Lee S, Sung S, Yoon T. The Survey of Mental Disorders in Korea. Sejong: Ministry of Health \& Welfare; 2017.

21. Cho MJ, Hahm BJ, Suh DW, Hong JP, Bae JN, Kim JK, et al. Development of a Korean version of the Composite International Diagnostic Interview (K-CIDI). J Korean Neuropsychiatr Assoc 2002;41:123-137.

22. Weisner C, Greenfield T, Room R. Trends in the treatment of alcohol problems in the US general population, 1979 through 1990. Am J Public Health 1995;85:55-60.

23. Kerr WC, Stockwell T. Understanding standard drinks and drinking guidelines. Drug Alcohol Rev 2012;31:200-205.

24. Spinelli C, Thyer BA. Is recovery from alcoholism without treatment possible? A review of the literature. Alcohol Treat Q 2017;35:426-444.

25. Ko S, Sohn A. Behaviors and culture of drinking among Korean people. Iran J Public Health 2018;47:47-56.

26. Jang JY, Kim DJ. Epidemiology of alcohol liver disease in Korea. Clin Mol Hepatol 2018;24:93-99.

27. Lee DW, Park HS, Lee TS, Kim MK, Kim YH. Korean working adults' and undergraduates' attitudes towards, and self-efficacy in, joining drinking parties. Soc Behav Pers 2006;34:487-498.
28. Health at a glance: Asia/Pacific 2012. Available at: http://www.oecd-ilibrary.org/social-issues-migration-health/health-at-a-glance-asia-pacific-2012_9789264183902-en. Accessed November, 1, 2020.

29. Dawson DA. Gender differences in the probability of alcohol treatment. J Subst Abus 1996;8:211-225.

30. Greenfield SF, Brooks AJ, Gordon SM, Green CA, Kropp F, McHugh RK, et al. Substance abuse treatment entry, retention, and outcome in women: a review of the literature. Drug Alcohol Depend 2007;86:1-21.

31. Bourdon JL, Tillman R, Francis MW, Dick DM, Stephenson M, Kamarajan $\mathrm{C}$, et al. Characterization of service use for alcohol problems across generations and sex in adults with alcohol use disorder. Alcohol Clin Exp Res 2020;44:746-757.

32. Grosso JA, Epstein EE, McCrady BS, Gaba A, Cook S, Backer-Fulghum LM, et al. Women's motivators for seeking treatment for alcohol use disorders. Addict Behav 2013;38:2236-2245.

33. Brady TM, Ashley OS. Women in Substance Abuse Treatment: Results from the Alcohol and Drug Services Study (ADSS). Rockville, MD: Substance Abuse and Mental Health Services Administration, Office of Applied Studies; 2005.

34. Covington S. Helping Women Recover: Creating Gender-Responsive Treatment. In: Straussner, SLA, Brown S, Editors. The Handbook of Addiction Treatment for Women: Theory and Practice. San Francisco, CA: Jossey-Bass; 2002, p. 52-72.

35. Prochaska JJ. Failure to treat tobacco use in mental health and addiction treatment settings: a form of harm reduction? Drug Alcohol Depend 2010;110:177-182.

36. McKelvey K, Thrul J, Ramo D. Impact of quitting smoking and smoking cessation treatment on substance use outcomes: An updated and narrative review. Addict Behav 2017;65:161-170.

37. Kahler CW, Borland R, Hyland A, McKee SA, O’Connor RJ, Fong GT, et al. Quitting smoking and change in alcohol consumption in the International Tobacco Control (ITC) Four Country Survey. Drug Alcohol Depend 2010;110:101-107.

38. Kim SJ, Chae W, Park WH, Park MH, Park EC, Jang SI. The impact of smoking cessation attempts on stress levels. BMC Public Health 2019; 19:267.

39. Berrington de Gonzalez A, Hartge P, Cerhan JR, Flint AJ, Hannan L, MacInnis RJ, et al. 2010. Body-mass index and mortality among 1.46 million white adults. N Engl J Med 2010;363:2211-2219.

40. Ciao AC, Latner JD, Durso LE. Treatment seeking and barriers to weight loss treatments of different intensity levels among obese and overweight individuals. Eat Weight Disord 2012;17:e9-e16.

41. Simpson CA, Tucker JA. Temporal sequencing of alcohol-related problems, problem recognition, and help-seeking episodes. Addict Behav 2004:27:659-674.

42. Weisner C, Matzger H. Missed opportunities in addressing drinking behavior in medical and mental health services. Alcohol Clin Exp Res 2003;27:1132-1141.

43. Castillo-Carniglia A, Keyes KM, Hasin DS, Cerdá M. Psychiatric comorbidities in alcohol use disorder. Lancet Psychiatry 2019;6:1068-1080.

44. Hajema KJ, Knibbe RA, Drop MJ. Social resources and alcohol related losses as predictors of help-seeking among male problem drinkers. J Stud Alcohol 1999;60:120-129.

45. Wu L, Ringwalt CL. Alcohol dependence and use of treatment services among women in the community. Am J Psychiatry 2004;161:1790-1797. 